\title{
Power allocation game for fading MIMO multiple access channels with antenna correlation
}

\author{
Samson Lasaulce \\ Lab. des Signaux et Systemes \\ CNRS - Supelec - Paris 11 \\ 91190, Gif-sur-Yvette, France \\ lasaulce@lss.supelec.fr
}

\author{
Alberto Suárez \\ Dpt. Communications Mobiles \\ Institut Eurecom \\ 06904, Sophia Antipolis, \\ France \\ suarezr@eurecom.fr \\ Laura Cottatellucci \\ Dpt. Communications Mobiles \\ Institut Eurecom \\ 06904, Sophia Antipolis, \\ France \\ cottatellucci@eurecom.fr
}

\author{
Merouane Debbah ${ }^{\dagger}$ \\ Dpt. Telecoms \\ Supelec \\ 91190, Gif-sur-Yvette, France \\ merouane.debbah@supelec.fr
}

\begin{abstract}
In this contribution, a power allocation game for multiple input multiple output multiple access channels is provided. Considering competing transmitting users, equipped with several antennas each and common multiple antennas at the receiver (base station), a game theoretic framework is conducted to analyze the optimum precoding matrices (power allocation and eigenvector transmit structure) such that each user maximizes selfishly his own rate under a power constraint (assuming single user decoding at the receiver). Interestingly, as the dimensions of the system grow i.e the numbers of transmitting and receiving antennas go to infinity but their ratio stays constant, a Nash equilibrium is shown to exist and be unique. The results are based on random matrix theory and provide, in the asymptotic case, a closed-form expression of the Nash equilibrium operating point. Each terminal can compute the power allocation independently based only on the knowledge of the statistics of the channel (spatial correlation structure at the transmitter and the receiver) and not its instantaneous realizations. This reduces dramatically the downlink overhead signaling protocol, which becomes important as the number of users grow. The asymptotic claims are then validated through simulations using only a finite number of antennas.
\end{abstract}

\footnotetext{
${ }^{*}$ This work is supported in part by the ANR project WINEM.

${ }^{\dagger}$ This work was supported by Alcatel-Lucent within the Alcatel-Lucent chair on flexible radio at Supelec.
}

Permission to make digital or hard copies of all or part of this work for personal or classroom use is granted without fee provided that copies are not made or distributed for profit or commercial advantage and that copies bear this notice and the full citation on the first page. To copy otherwise, to republish, to post on servers or to redistribute to lists, requires prior specific permission and/or a fee.

GameComm '07, October 22, 2007, Nantes, France

Copyright 2007 ICST 978-963-9799-00-4.

\section{Categories and Subject Descriptors}

[Game theory for communications networks]: miscellaneous; d.2.8 [Power control games]: metrics-Complexity measures, performance measures

\section{General Terms}

Random matrix theory, Game theory, Information theory.

\section{Keywords}

Multiple access channel, MIMO systems, antenna correlation, power allocation game, Nash equilibrium.

\section{INTRODUCTION}

We consider the uplink of a typical cellular system, which is a multiple access channel (MAC). The MAC consists of several users, called mobile stations in cellular systems, sending independent messages to a common receiver, called the base station. More specifically we assume that both the base and mobile stations are equipped with - possibly correlatedmultiple antennas and the different links into play are fading channels. In this context we want to investigate the optimum power allocation issue at the mobile stations in the case where the base station does not send any control signal to the mobile stations.

From an information theoretic point of view, the optimal power and rate policies for the fading single input single output (SISO) MAC have been determined by [1], which leads to the MAC ergodic capacity region. Recently, the authors of [2][3] addressed the fading multiple input multiple output (MIMO) MAC with correlated antennas and covariance feedback at the transmitters and determined the optimum power allocation policy in terms of ergodic sum capacity. We consider the same framework as [2][3] but we do not assume the power allocation policies to be centralized. In order to reduce the signaling protocol overhead, we want to determine the best distributed power allocation strategies: each user wants selfishly to maximize its own utility instead of a global utility function such as the sum-capacity. A convenient tool to address this kind of problems turns out to be 
game theory [4][5]. In this respect the authors of [6] used a game theoretic approach to characterize the information rates of the fading SISO and single input multiple output (SIMO) multiple access channels. Note that different utility functions can be defined such as those maximizing energyefficiency (see e.g. [7][8]). In this paper the individual utility function we selected is the average mutual information between the transmitted signal of the considered user and the signal received by the base station. In [9], which is the closest work to the one presented here, the authors have chosen the individual mutual information for studying static MIMO MAC channels when the transmitters have perfect channel state information (CSI). Here, given the fact that the channel is time-varying, we consider the ergodic mutual information, which does not depend on a particular realization of the channel but on its statistics. Therefore our work differs from [9] in at least three important points:

- (a) each user is only informed with the statistics of its own channel and not with its instantaneous knowledge;

- (b) the transmit and receive antennas can be correlated (this feature cannot be considered when assuming perfect CSI since each transmitter exploits the realization of the channel itself);

- (c) we use the theory of random matrices to simplify the design of the power allocation algorithms.

Concerning the latter point, random matrix theory will be used with the same approach as the authors of [10], who studied the impact of antenna correlation on fading MIMO single-user channels. As it will be seen, it considerably simplifies the derivation of distributed power allocation algorithms and the analysis of their properties.

This paper is structured as follows. We first provide the signal model used to represent the fading MIMO MAC channel with antenna correlations (section 2). After defining and justifying the utility function for the users in section 3 , we provide the corresponding optimum power allocation scheme in section 4. Sufficient conditions for the existence and uniqueness of a Nash equilibrium are provided in section 4.3. Section 5 provides simulation results that show the benefits of using the proposed power allocation policy instead of allocating the available transmit power uniformly over the different transmit antennas (isotropic power allocation).

\section{SYSTEM MODEL}

Notations: in this paper, the notations $s, \underline{v}, \mathbf{M}$ stand for scalar, vector and matrix respectively. The superscripts $(.)^{T}$ and $(.)^{H}$ denote transpose and transpose conjugate, respectively. The trace of the matrix $\mathbf{M}$ is denoted by $\operatorname{Tr}(\mathbf{M})$. The mathematical expectation operator is denoted by $\mathbb{E}($.$) .$ $\Re$ and $\Im$ denote respectively the real and imaginary parts. $\mathcal{N}(\underline{v}, \mathbf{M})$ denotes the complex Gaussian distribution with mean $\underline{v}$ and covariance $\mathbf{M}$.

We consider the uplink of a single cell with $K$ active users (see figure 1). Each mobile station is equipped with $n_{t}$ antennas whereas the base station has $n_{r}$ antennas (thus we assume the same number of transmitting antennas for all the users). In our analysis the flat fading channel matrices of the different links can possibly vary from symbol vector (or space-time codeword) to symbol vector (or space-time codeword). We assume that the receiver knows all the channel matrices (coherent communication assumption) whereas each transmitter has only access to the statistics of the channel over which it sends a message. The equivalent baseband signal received by the base station can be written as

$$
\underline{y}(\tau)=\sum_{k=1}^{K} \boldsymbol{H}_{k}(\tau) \underline{x}_{k}(\tau)+\underline{n}(\tau)
$$

where $\underline{x}_{k}(\tau)$ is the $n_{t}$-dimensional column vector of symbols transmitted by user $k$ at time $\tau, \mathbf{H}_{k}(\tau) \in \mathbb{C}^{n_{r} \times n_{t}}$ is the channel matrix (stationary and ergodic process) of user $k$ and $n(\tau)$ is a $n_{t}$-dimensional complex white Gaussian noise distributed as $\mathcal{N}\left(\underline{0}, \sigma^{2} \mathbf{I}_{r}\right)$. For simplicity we will omit the time index $\tau$ from our notations. Each channel input is subject to a power constraint $\operatorname{Tr}\left[\mathbb{E}\left(\underline{x}_{k} \underline{x}_{k}^{H}\right)\right] \triangleq \operatorname{Tr}\left(\mathbf{Q}_{k}\right) \leq n_{t}$. Here we implicitly assume that the mobile terminals have the same transmit power, which is a reasonable assumption in a cellular system.

In order to take into account the antenna correlation effects at the transmitters and receiver we will assume the different channel matrices to be structured according to the Kronecker propagation model [11]:

$$
\forall k \in\{1, \ldots, K\}, \mathbf{H}_{k}=\mathbf{R}^{\frac{1}{2}} \boldsymbol{\Theta}_{k} \mathbf{T}_{k}^{\frac{1}{2}}
$$

where $\mathbf{R}$ is the receive antenna correlation matrix, $\mathbf{T}_{k}$ is the transmit antenna correlation matrix for user $k$ and $\boldsymbol{\Theta}_{k}$ is an $n_{t} \times n_{t}$ matrix whose entries are zero-mean independent and identically distributed (i.i.d.) Gaussian random variables with variance $\frac{1}{n_{t}}$. It turns out that in cellular systems the Kronecker model can often be simplified because the receive antennas are sufficiently spaced such as there is no correlation at the receiver side, which implies that $\mathbf{R}=\mathbf{I}$. However our analysis can be extended to the case of receive correlation by using similar arguments to those of [3][12].

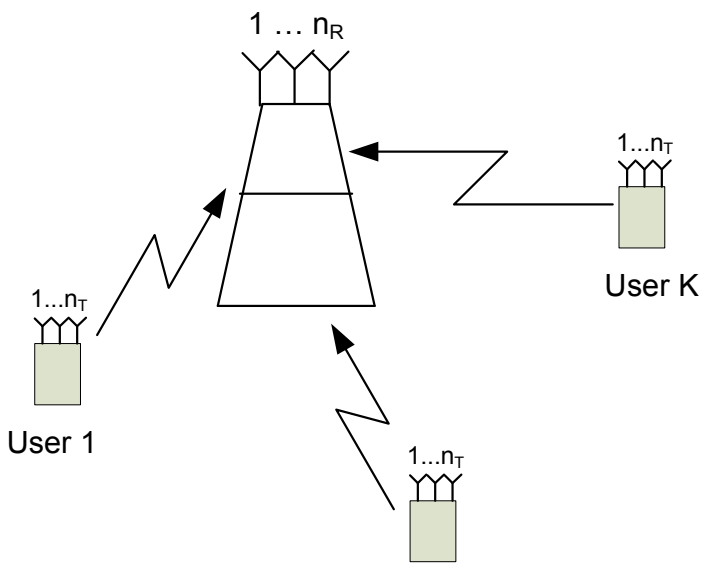

User 2

Figure 1: System under investigation: $\mathrm{K}$ users with $n_{T}$ antennas each, and a BS with $n_{R}$ receive antennas

\section{PROBLEM STATEMENT}

In this section, we first justify the choice of the considered utility functions (subsection 3.1). Then, in subsection 
3.2 we show that the search for the input covariance matrix maximizing the utility function of a given user can be simplified. In fact, without loss of optimality, we can impose a certain structure on the eigenvectors of the input covariance matrix. Then (section 4), based on this choice for the eigenvectors, we use random matrix theory to obtain an accurate and simple approximation of the utility function, which allows us to maximize it and obtain the optimum eigenvalues of the input covariance matrix.

\subsection{On the choice of the utility function}

As mentioned in the introduction, instead of maximizing the sum-capacity [3] each user wants to selfishly maximize his non-cooperative utility function. Therefore, assuming single-user decoding at the base station the information rate achieved by user $k$ equals the mutual information between $\underline{x}_{k}$ and $y$ conditioned on the overall channel matrix $\mathbf{H}=$ $\left[\mathbf{H}_{1} \mathbf{H}_{2} \ldots \mathbf{H}_{K}\right]$ :

$$
\begin{aligned}
I\left(\underline{x}_{k} ; \underline{y} \mid \mathbf{H}\right)= & \mathbb{E}\left[\log _{2} \frac{\left|\sum_{\ell} \mathbf{H}_{\ell} \mathbf{Q}_{\ell} \mathbf{H}_{\ell}^{H}+\sigma^{2} \mathbf{I}\right|}{\left|\sum_{\ell \neq k} \mathbf{H}_{\ell} \mathbf{Q}_{\ell} \mathbf{H}_{\ell}^{H}+\sigma^{2} \mathbf{I}\right|}\right] \\
= & \mathbb{E}\left[\log _{2}\left|\sum_{\ell} \mathbf{H}_{\ell} \mathbf{Q}_{\ell} \mathbf{H}_{\ell}^{H}+\sigma^{2} \mathbf{I}\right|\right] \\
& -\mathbb{E}\left[\log _{2}\left|\sum_{\ell \neq k} \mathbf{H}_{\ell} \mathbf{Q}_{\ell} \mathbf{H}_{\ell}^{H}+\sigma^{2} \mathbf{I}\right|\right] \\
\triangleq & C_{k}^{(1)}-\mathbb{E}\left[\log _{2}\left|\sum_{\ell \neq k} \mathbf{H}_{\ell} \mathbf{Q}_{\ell} \mathbf{H}_{\ell}^{H}+\sigma^{2} \mathbf{I}\right|\right]
\end{aligned}
$$

where $|\mathbf{A}|$ stands for the determinant of the matrix $\mathbf{A}$ and the expectation is taken over the random channel matrices $\mathbf{H}_{1}, \ldots, \mathbf{H}_{K}$. We see that the second term of the ergodic mutual information (EMI) does not depend on the input covariance matrix of user $k$ and therefore does not need to be considered for the individual utility function of the different users $k \in\{1, \ldots, K\}$, which will be chosen then as

$$
C_{k}^{(1)}\left(\mathbf{Q}_{k}\right)=\mathbb{E}\left[\log _{2}\left|\sum_{\ell=1}^{K} \mathbf{H}_{\ell} \mathbf{Q}_{\ell} \mathbf{H}_{\ell}^{H}+\sigma^{2} \mathbf{I}\right|\right] .
$$

Note that, for the centralized approach, maximizing the EMI with respect to the input covariance matrix $\mathbf{Q}_{k}=\mathbb{E}\left(\underline{x}_{k} \underline{x}_{k}^{H}\right)$ leads to the channel/Shannon capacity for fast fading MIMO channels i.e. when the channel vary from symbol to symbol. This capacity is achieved by averaging over channel variations over time. For slow fading MIMO channels, i.e. when the channel matrix remains constant over a certain block duration much smaller than the channel coherence time, no such averaging is possible and one has to communicate at rates smaller than the ergodic capacity. The maximum EMI is therefore a rate upper bound for slow fading MIMO channels and only a fraction of it can be achieved $^{1}$. A more suited performance metric to study slow-fading channels is the outage capacity, which is related to the variance of the mutual information instead of its mean (EMI) as we do here. This can be considered as a possible extension of the present work.

\footnotetext{
${ }^{1}$ This fraction is called the multiplexing gain in [13] where the authors introduced the famous diversity multiplexing trade-off.
}

Now we have justified why we chose $C_{k}^{(1)}\left(\mathbf{Q}_{k}\right)$ for the utility function, we address, for each user, the optimization problem of this function with respect to $\mathbf{Q}_{k}$ under the individual power constraint $\operatorname{Tr}\left(\mathbf{Q}_{k}\right) \leq n_{t}$. The input covariance matrix can be decomposed spectrally as: $\mathbf{Q}_{k}=\mathbf{V}_{k} \mathbf{P}_{k} \mathbf{V}_{k}^{H}$, where $\mathbf{P}_{k}=\operatorname{Diag}\left(P_{k}(1), \ldots, P_{k}\left(n_{t}\right)\right)$. The purpose of the following sections is precisely to show how to determine the eigenvectors and eigenvalues of $\mathbf{Q}_{k}$ in a game theoretic setting.

\subsection{Optimum eigenvectors of the input covari- ance matrix}

The utility function we are interested in is:

$$
C_{k}^{(1)}\left(\mathbf{Q}_{k}\right)=\mathbb{E}\left[\log _{2}\left|\sum_{\ell=1}^{K} \mathbf{H}_{\ell} \mathbf{Q}_{\ell} \mathbf{H}_{\ell}^{H}+\sigma^{2} \mathbf{I}\right|\right] .
$$

Indeed, the second term of $C_{k}$ does not depend on $\mathbf{Q}_{k}$, which means it does not need to be considered. Now remind the ergodic sum capacity expression for the MIMO multiple access channel

$$
C_{\text {sum }}=\max _{\mathbf{Q}_{1}, \ldots, \mathbf{Q}_{K}} \mathbb{E}\left[\log _{2} \frac{\left|\sum_{\ell=1}^{K} \mathbf{H}_{\ell} \mathbf{Q}_{\ell} \mathbf{H}_{\ell}^{H}+\sigma^{2} \mathbf{I}\right|}{\left|\sigma^{2} \mathbf{I}\right|}\right]
$$

under the classical trace constraints. So, up to an additive constant term we want to optimize the same function as in [3]. The difference is that we only optimize it over $\mathbf{Q}_{k}$ instead over $\left(\mathbf{Q}_{1}, \ldots, \mathbf{Q}_{K}\right)$. Therefore the proof of [3] can be reused to assert that there is no loss in restricting the search for the optimum covariance matrix by imposing the structure $\mathbf{Q}_{k}=\mathbf{U}_{k} \boldsymbol{\Lambda}_{k} \mathbf{U}_{k}^{H}$ where $\mathbf{T}_{k}=\mathbf{U}_{k} \mathbf{D}_{k} \mathbf{U}_{k}^{H}$, in other words the transmit eigenvectors match the transmit correlation matrix. In order to make this paper sufficiently selfcontained we will provide the proof of the following lemma:

Lemma 1 (Optimum eigenvectors). Let $\mathcal{Q}$ be the set of $n_{t} \times n_{t}$ Hermitian matrices such that $\operatorname{Tr}(\boldsymbol{Q}) \leq n_{t}$ i.e. $\mathcal{Q}=$ $\left\{\left(\boldsymbol{Q}_{1}, \ldots \boldsymbol{Q}_{K}\right): \forall k \in\{1, \ldots, K\}, \boldsymbol{Q}_{k}=\boldsymbol{Q}_{k}^{H}, \operatorname{Tr}\left(\boldsymbol{Q}_{k}\right) \leq n_{t}\right\}$ Additionally, let $\mathcal{S}$ be the subset of $\mathcal{Q}$ such that $\boldsymbol{Q}_{k}=\bar{U}_{k} \boldsymbol{P}_{k} \boldsymbol{U}_{k}^{H}$ where $\mathbf{U}_{k}$ represents the eigenvectors of $\mathbf{T}_{k}$. Then

$$
\max _{\mathbf{Q}_{k} \in \mathcal{Q}} C_{k}^{(1)}=\max _{\mathbf{Q}_{k} \in \mathcal{S}} C_{k}^{(1)}
$$

Proof. By definition: $\mathbf{H}_{\ell}=\boldsymbol{\Theta}_{\ell} \mathbf{T}_{\ell}^{\frac{1}{2}}=\boldsymbol{\Theta}_{\ell} \mathbf{U}_{\ell} \mathbf{D}_{\ell}^{\frac{1}{2}} \mathbf{U}_{\ell}^{H}$, where $\boldsymbol{\Theta}_{\ell}$ is a zero-mean i.i.d. Gaussian identity covariance random matrix. Using the fact that multiplying $\boldsymbol{\Theta}_{\ell}$ by a unitary matrix does not change its joint distribution one can write:

$$
\begin{aligned}
& \max _{\mathbf{Q}_{k}} C_{k}^{(1)}= \\
& \max _{\mathbf{Q}_{k}}\left[\log _{2}\left|\sum_{\ell=1}^{K} \boldsymbol{\Theta}_{\ell} \mathbf{U}_{\ell} \mathbf{D}_{\ell}^{\frac{1}{2}} \mathbf{U}_{\ell}^{H} \mathbf{Q}_{\ell} \mathbf{U}_{\ell} \mathbf{D}_{\ell}^{\frac{1}{2}} \mathbf{U}_{\ell}^{H} \boldsymbol{\Theta}_{\ell}^{H}+\sigma^{2} \mathbf{I}\right|\right]= \\
& \max _{\mathbf{Q}_{k}}\left[\log _{2}\left|\sum_{\ell=1}^{K} \boldsymbol{\Theta}_{\ell} \mathbf{D}_{\ell}^{\frac{1}{2}} \mathbf{U}_{\ell}^{H} \mathbf{Q}_{\ell} \mathbf{U}_{\ell} \mathbf{D}_{\ell}^{\frac{1}{2}} \mathbf{\Theta}_{\ell}^{H}+\sigma^{2} \mathbf{I}\right|\right]
\end{aligned}
$$

Then we can spectrally decompose the matrix $\mathbf{D}_{\ell}^{\frac{1}{2}} \mathbf{U}_{\ell}^{H} \mathbf{Q}_{\ell} \mathbf{U}_{\ell} \mathbf{D}_{\ell}^{\frac{1}{2}}=$ 
$\tilde{\mathbf{U}}_{\ell} \tilde{\mathbf{D}}_{\ell} \tilde{\mathbf{U}}_{\ell}^{H}$ and write that

$$
\begin{aligned}
\max _{\mathbf{Q}_{k}} C_{k}^{(1)} & =\max _{\mathbf{Q}_{k}} \mathbb{E}\left[\log _{2}\left|\sum_{\ell=1}^{K} \boldsymbol{\Theta}_{\ell} \tilde{\mathbf{U}}_{\ell} \tilde{\mathbf{D}}_{\ell} \tilde{\mathbf{U}}_{\ell}^{H} \mathbf{\Theta}_{\ell}^{H}+\sigma^{2} \mathbf{I}\right|\right] \\
& =\max _{\mathbf{Q}_{k}} \mathbb{E}\left[\log _{2}\left|\sum_{\ell=1}^{K} \boldsymbol{\Theta}_{\ell} \tilde{\mathbf{D}}_{\ell} \boldsymbol{\Theta}_{\ell}^{H}+\sigma^{2} \mathbf{I}\right|\right]
\end{aligned}
$$

We see that the function to be optimized depends on the eigenvectors $\tilde{\mathbf{U}}_{k}$ only through the power constraint:

$$
\operatorname{Tr}\left(\mathbf{Q}_{\mathrm{k}}\right)=\operatorname{Tr}\left(\tilde{\mathbf{U}}_{\mathrm{k}}^{\mathrm{H}} \mathbf{D}_{\mathrm{k}}^{-1} \tilde{\mathbf{U}}_{\mathrm{k}} \tilde{\mathbf{D}}_{\mathrm{k}}\right) \leq \mathrm{n}_{\mathrm{t}} .
$$

The matrix $\tilde{\mathbf{U}}_{k}$ can be chosen arbitrarily provided it meets the power constraint $\operatorname{Tr}\left(\mathbf{Q}_{\mathbf{k}}\right) \leq \mathrm{n}_{\mathrm{t}}$. The choice $\tilde{\mathbf{U}}_{k}=\mathbf{I}$ is feasible since $\operatorname{Tr}\left(\mathbf{D}_{\mathrm{k}}^{-1} \tilde{\mathbf{D}}_{\mathrm{k}}\right) \leq \operatorname{Tr}\left(\tilde{\mathbf{U}}_{\mathrm{k}}^{\mathrm{H}} \mathbf{D}_{\mathrm{k}}^{-1} \tilde{\mathbf{U}}_{\mathrm{k}} \tilde{\mathbf{D}}_{\mathrm{k}}\right) \leq \mathrm{n}_{\mathrm{t}}$. This shows that $\mathbf{Q}_{k}$ can be chosen without loss of optimality to be structured as:

$$
\mathbf{Q}_{k}=\mathbf{U}_{k} \mathbf{D}_{k}^{-1} \tilde{\mathbf{D}}_{k} \mathbf{U}_{k}^{H}
$$

Quite interestingly, if each user wants to maximize his own rate, each user will independently choose an eigenvector basis which matches his transmit correlation matrix (and does not depend on the channels of all the other users). This is a quite important result when considering the decentralized approach as it reduces the power allocation game algorithm to the choice of the transmit powers only. As we will show in the next section, the transmit power allocation algorithm will also be simplified as it will turn out to be independent of the channel realizations but will only depend on the statistics of the different channels.

\section{OPTIMUM SELFISH POWER ALLOCATION POLICY: A LARGE SYSTEM APPROACH}

\subsection{Asymptotic expression of the utility func- tion}

In the previous section we have shown that, for a given user, there is no loss of optimality by choosing the eigenvectors of $\mathbf{Q}_{k}$ to be equal to those of the transmit correlation matrix $\mathbf{T}_{k}=\mathbf{U}_{k} \mathbf{D}_{k} \mathbf{U}_{k}^{H}$. As a consequence, one can exploit the asymptotic results of [14] derived for fading MIMO single-user channels with transmit and receive antenna correlation for large systems. Indeed, from now on, we assume the asymptotic regime in terms of the number of antennas, which is defined as:

- $n_{t} \rightarrow \infty$;

- $n_{r} \rightarrow \infty$;

- $\lim _{n_{t} \rightarrow \infty, n_{r} \rightarrow \infty} \frac{n_{t}}{n_{r}}=c$ where $0<c<\infty$.

For each user $k \in\{1, \ldots, K\}$, we also suppose that $d_{k}(1), \ldots, d_{k}\left(n_{t}\right)$ which are the elements of the diagonal matrix $\mathbf{D}_{k}$, have an empirical distribution that converges to a probability density function $f_{k}(t)$ i.e.

$$
\frac{1}{n_{t}} \sum_{i=1}^{n_{t}} \delta\left(t-d_{k}(i)\right) \rightarrow f_{k}(t) .
$$

Under these assumptions, one can apply Theorem 3.7 of [14] and verify that $C_{k}^{(1)}$ can be approximated by $\bar{C}_{k}^{(1)}$ :

$$
\begin{aligned}
\bar{C}_{k}^{(1)}= & \frac{1}{n_{r}} \sum_{\ell=1}^{K} \sum_{i=1}^{n_{t}} \log _{2}\left[1+K \rho P_{\ell}(i) d_{\ell}(i) \alpha\right]+\log _{2}(1+K \rho \beta) \\
& -\frac{n_{t} K^{2}}{n_{r}} \rho \alpha \beta \log _{2} e
\end{aligned}
$$

where $\rho \triangleq \frac{1}{\sigma^{2}}$ and the pair $(\alpha, \beta)$ is determined by the following system of equations:

$$
\left\{\begin{aligned}
\alpha & =\frac{n_{r}}{K n_{t}} \frac{1}{1+K \rho \beta} \\
\beta & =\frac{1}{K n_{t}} \sum_{\ell=1}^{K} \sum_{i=1}^{n_{t}} \frac{P_{\ell}(i) d_{\ell}(i)}{1+K \rho P_{\ell}(i) d_{\ell}(i) \alpha} .
\end{aligned}\right.
$$

Note that in order to make this approximation accurate the numbers of antennas do not need to be very high. This is especially true when the metric of interest is the ergodic mutual information since one benefits from a double averaging effect, one from the randomness of the matrices into play and the other one from the expectation operator. For example, in [12], the asymptotic regime is shown to be reached with a very small relative error for a $4 \times 4$ fading MIMO single-user system.

\subsection{Power allocation algorithm}

For each user $k$, we want to determine the optimal way, in the sense of his approximated utility function $\bar{C}_{k}^{(1)}$, to share its available power between the transmit eigenmodes. To solve this constrained optimized problem we introduce the Lagrange multiplier $\lambda_{k}$ and define the function

$$
\mathcal{L}_{\lambda_{k}}\left(P_{k}(i)\right) \triangleq \bar{C}_{k}^{(1)}-\lambda_{k} \times\left(\sum_{j=1}^{n_{t}} P_{k}(j)-n_{t}\right)
$$

and search for the solution(s) $P_{k}^{*}(i)$ such that $\frac{\partial \mathcal{L}_{\lambda_{k}}}{\partial P_{k}(i)}=0$. The solution of the corresponding optimization problem is stated through the following lemma.

Lemma 2 (Optimum eigenvalues). Assume that the pair $(\alpha, \beta)$ is a solution of the system of equations (14). Then the spatial power allocation maximizing the constrained approximated utility function (15) is given by the following water-filling solution:

$$
P_{k}^{*}(i)=\left[\frac{1}{n_{r} \ln 2 \lambda_{k}}-\frac{1}{K \rho d_{k}(i) \alpha}\right]^{+}
$$

where we used the notation $[x]^{+}=\max (x, 0)$.

Proof. To obtain the water-filling solution(s) one first needs to explicit $\frac{\partial \bar{C}_{k}^{(1)}}{\partial P_{k}(i)}$, which is the purpose of the following calculation. The main interest in the proposed derivation is that one does not need to assume $\alpha$ or $\beta$ to be independent of $P_{k}(i)$, which avoids one to study the convergence issues for $\alpha$ and $\beta$. Otherwise, if they are assumed to be strict , constants with respect to a given power $P_{k}(i)$, the result can be obtained much more easily. We want to prove that the derivative of the approximated utility function of user $k$ can be expressed as:

$$
\frac{\partial \bar{C}_{k}^{(1)}}{\partial P_{k}(i)}=\frac{1}{n_{r} \ln 2} \frac{K \rho d_{k}(i) \alpha}{1+K \rho P_{k}(i) d_{k}(i) \alpha} .
$$


We have:

$$
\begin{aligned}
n_{r} \bar{C}_{k}^{(1)} & =\log _{2}\left\{\prod_{\ell, j}\left[1+K \rho P_{\ell}(j) d_{\ell}(j) \alpha\left(P_{k}(i)\right)\right] \times\right. \\
& \left.\times\left(1+K \rho \beta\left(P_{k}(i)\right)\right)^{n_{r}} e^{-n_{t} K^{2} \rho \alpha\left(P_{k}(i)\right) \beta\left(P_{k}(i)\right)}\right\}
\end{aligned}
$$

Define $U \triangleq \prod_{\ell, j}\left[1+K \rho P_{\ell}(j) d_{\ell}(j) \alpha\left(P_{k}(i)\right)\right]$

$V \triangleq\left(1+K \rho \beta\left(P_{k}(i)\right)\right)^{n_{r}} e^{-n_{t} K^{2} \rho \alpha\left(P_{k}(i)\right) \beta\left(P_{k}(i)\right)}$. With these notations:

$$
\frac{\partial n_{r} \bar{C}_{k}^{(1)}}{\partial P_{k}(i)}=\frac{1}{U V} \frac{\partial U V}{\partial P_{k}(i)}
$$

It turns out that $\frac{\partial U V}{\partial P_{k}(i)}=U V \times \frac{K \rho d_{k}(i) \alpha}{1+K \rho P_{k}(i) d_{k}(i) \alpha}$. This is what we want to show.

First step:

$$
\begin{aligned}
U^{\prime} & \triangleq \frac{\partial U}{\partial P_{k}(i)} \\
& =\sum_{\ell, j} \prod_{\left(\ell^{\prime}, j^{\prime}\right) \neq(\ell, j)}\left[1+K \rho P_{\ell^{\prime}}\left(j^{\prime}\right) d_{\ell^{\prime}}\left(j^{\prime}\right) \alpha\right] \times \\
& \times\left[1+K \rho P_{\ell}(j) d_{\ell}(j) \alpha\right]^{\prime} \\
& =\sum_{\ell, j} \frac{N(\ell, j)}{1+K \rho P_{\ell}(j) d_{\ell}(j) \alpha} \underbrace{\prod_{\ell^{\prime}, j^{\prime}}\left[1+K \rho P_{\ell^{\prime}}\left(j^{\prime}\right) d_{\ell^{\prime}}\left(j^{\prime}\right) \alpha\right]}_{U}
\end{aligned}
$$

where

$$
N(\ell, j)=\mid \begin{array}{ll}
K \rho P_{\ell}(j) d_{\ell}(j) \alpha^{\prime} & \text { if }(\ell, j) \neq(k, i) \\
K \rho d_{k}(i)\left(\alpha+P_{k}(i) \alpha^{\prime}\right) & \text { if }(\ell, j)=(k, i)
\end{array}
$$

Second step:

$$
\begin{aligned}
V^{\prime} & \triangleq \frac{\partial V}{\partial P_{k}(i)} \\
& =V \times K \rho\left[\frac{n_{r} \beta^{\prime}}{1+K \rho \beta}-K n_{t}\left(\alpha^{\prime} \beta+\alpha \beta^{\prime}\right)\right] .
\end{aligned}
$$

From this we have that

$$
\begin{aligned}
\frac{\partial U V}{\partial P_{k}(i)} & =U^{\prime} V+U V^{\prime} \\
& =U V \times\left\{\sum_{\ell, j} \frac{N(\ell, j)}{1+K \rho P_{\ell}(j) d_{\ell}(j) \alpha}\right. \\
& \left.+K \rho\left[\frac{n_{r} \beta^{\prime}}{1+K \rho \beta}-K n_{t}\left(\alpha^{\prime} \beta+\alpha \beta^{\prime}\right)\right]\right\} \\
& =U V \times\left\{\sum_{(\ell, j) \neq(k, i)} \frac{K \rho P_{\ell}(j) d_{\ell}(j) \alpha^{\prime}}{1+K \rho P_{\ell}(j) d_{\ell}(j) \alpha}\right. \\
& +\frac{K \rho d_{k}(i)\left(\alpha+P_{k}(i) \alpha\right)^{\prime}}{1+K \rho P_{k}(i) d_{k}(i) \alpha} \\
& \left.+K \rho\left[\frac{n_{r} \beta^{\prime}}{1+K \rho \beta}-K n_{t}\left(\alpha^{\prime} \beta+\alpha \beta^{\prime}\right)\right]\right\}
\end{aligned}
$$

Now using the relations

$$
\left\{\begin{aligned}
\alpha & =\frac{n_{r}}{K n_{t}} \frac{1}{1+K \rho \beta} \\
\beta & =\frac{1}{K n_{t}} \sum_{\ell, j} \frac{P_{\ell}(j) d_{\ell}(j)}{1+K \rho P_{\ell}(j) d_{\ell}(j) \alpha} \\
\alpha^{\prime} & =\frac{K \rho \beta^{\prime}}{1+K \rho \beta} \alpha \\
\beta^{\prime} & =\frac{-n_{r}}{K n_{t} K \rho} \frac{1}{\alpha^{2}}
\end{aligned}\right.
$$

we find, after simplifications, the proposed expression for the derivative of $\bar{C}_{k}^{(1)}$.

Finally, by setting the derivative of $\mathcal{L}_{\lambda_{k}}\left(P_{k}(i)\right)$ to zero we find that:

$$
P_{k}^{*}(i)=\left[\frac{1}{n_{r} \ln 2 \lambda_{k}}-\frac{1}{K \rho d_{k}(i) \alpha}\right]^{+}
$$

In the water-filling procedure the Lagrangian multiplier $\lambda_{k}$, for user $k$, is tuned in order to meet the power constraint. In a finite setting the corresponding $\lambda_{k}$ would depend on the particular values of $d_{k}(i)$. However, in the asymptotic case, by assuming a known law $p_{k}$ for the diagonal terms $d_{k}(i)$, so that

$$
\frac{1}{n_{t}} \sum_{i=1}^{n_{t}} \frac{1}{d_{k}(i)} \rightarrow \int \frac{f_{k}(t)}{t}
$$

we see that $\lambda_{k}$ can be expressed analytically and only depends on the distribution of $d_{k}(i)$ according to the following relation:

$$
\begin{aligned}
1 & =\int_{0}^{+\infty}\left[\frac{1}{\mu_{k}}-\frac{1}{K \rho t \alpha}\right]^{+} f_{k}(t) d t \quad \text { where } \mu_{k}=n_{r} \ln 2 \lambda_{k} \\
& =\int_{\frac{\mu_{k}}{K \rho \alpha}}^{+\infty}\left(\frac{1}{\mu_{k}}-\frac{1}{K \rho t \alpha}\right) f_{k}(t) d t
\end{aligned}
$$

where $\mu_{k}$ is obtained through

$$
\mu_{k}=\frac{\int_{\frac{\mu_{k}}{K \rho \alpha}}^{+\infty} f_{k}(t) d t}{1+K \rho \alpha \int_{\frac{\mu_{k}}{K \rho \alpha}}^{+\infty} \frac{f_{k}(t)}{t} d t} .
$$

At this point we are in position to describe the proposed iterative power allocation algorithm:

1. Initialize $\alpha$ with a value in the interval $\left[\alpha_{\min }, \alpha_{\max }\right]$ with $\alpha_{\min }=\frac{n_{r}}{K n_{t}} \frac{1}{1+K \rho}$ and $\alpha_{\max }=\frac{n_{r}}{K n_{t}}$.

2. Apply water-filling over the $d_{k}(i)$ by using equation (16) in order to find $P_{k}(i)$ for all $i \in\left\{1, \ldots, n_{t}\right\}$ and $k \in\{1, \ldots, K\}$. For this purpose make use of equation (22) to find $\mu_{k}$ for all $k \in\{1, \ldots, K\}$.

3. By using the powers obtained at the previous step, update the value of $\alpha$ by searching for the solution of the system of equations (14).

4. If $\alpha$ has not converged (fix an arbitrary accuracy level on $\alpha$ ) go to step 1 . Otherwise stop the iterative procedure and go to step 5 . 
5. Apply for the last time the water-filling procedure over the $d_{k}(i)$ by using equation (16) in order to find $P_{k}(i)$ for all $i \in\left\{1, \ldots, n_{t}\right\}$ and $k \in\{1, \ldots, K\}$.

Depending on the assumptions one makes at the terminals, step 3 can slightly change. If the $K n_{t}$ correlation terms $d_{k}(i), k \in\{1, \ldots, K\}, i \in\left\{1, \ldots, n_{t}\right\}$ are assumed to be known at all transmitters step 3 can be implemented by using eq. (14). One can also restrict the knowledge of the transmitters to the probability density function $f_{k}(t), k \in\{1, \ldots, K\}$ in which case one just needs to use equation given below for step 3. Indeed, in the asymptotic regime, the existence and uniqueness of the solution to the proposed optimization procedure is related to the existence and uniqueness of the solution $\alpha$, which verifies the following key equation:

$$
K c \alpha+\frac{1}{K} \sum_{\ell=1}^{K} \int_{\frac{\mu_{\ell}}{K \rho \alpha}}^{+\infty}\left(1-\frac{\mu_{\ell}}{K \rho \alpha t}\right) f_{\ell}(t) d t=1 .
$$

This equation is obtained eliminating $\beta$ in the system of equations (14), using the fact that $\frac{d_{\ell}(i)}{1+K \rho P_{\ell}(i) d_{\ell}(i) \alpha}=\mu_{\ell}$ (equation (17)) when a non-zero power is allocated to the $i^{\text {th }}$ antenna, using the expression of $P_{\ell}^{*}(i)$ (equation (19)) and using the probability density function $f_{k}(t)$ instead of $d_{k}(i)$ in the asymptotic regime. Studying the existence and uniqueness of the solution of this equation is the purpose of the next section.

\subsection{Nash equilibrium}

For the non-cooperative power allocation game proposed in this paper, a Nash equilibrium is a set of power vectors $\left\{\underline{P}_{1}^{*}, \ldots, P_{K}^{*}\right\}$, with $\forall k \in\{1, \ldots, K\}, \underline{P}_{k}^{*}=\left(P_{k}^{*}(1), \ldots, P_{k}^{*}\left(n_{t}\right)\right)$ such that no user can unilaterally improve its utility by choosing a different power vector i.e. $\left\{\underline{P}_{1}^{*}, \ldots, \underline{P}_{K}^{*}\right\}$ is a Nash equilibrium if and only if:

$$
\bar{C}_{k}^{(1)}\left(\underline{P}_{k}^{*}, \underline{P}_{-k}^{*}\right) \geq \bar{C}_{k}^{(1)}\left(\underline{P}_{k}, \underline{P}_{-k}^{*}\right) \text { for all } \underline{P}_{k}
$$

where $\underline{P}_{-k}^{*}$ stands for the strategies of all the users except for user $k$.

In this section we investigate the existence and uniqueness of a Nash equilibrium when all the active users perform allocation power according to the proposed iterative algorithm. In order to prove the existence and uniqueness of a Nash equilibrium one needs to study the convergence of this algorithm. In the literature of random matrix theory this kind of algorithm is quite common (see e.g. [10], [12]). Although many simulation results illustrate the convergence of these algorithms, the convergence proof has only been provided very recently in [17]. ${ }^{2}$ The authors proved the convergence of this algorithm in the case of Rician MIMO single-user channels with two-sided antenna correlation. By noticing that maximizing the utility function of user $k$ used in this paper amounts to maximizing the EMI of a $K n_{t} \times n_{r}$ MIMO system with respect to $\mathbf{Q}_{k}$ one can therefore apply the results of [17]. First these results allow us to assert the strict concavity of the asymptotic approximant of $I\left(\underline{x}_{1}, \ldots, \underline{x}_{K} ; \underline{y} \mid \mathbf{H}\right)$ with respect to $\left(\mathbf{Q}_{1}, \ldots, \mathbf{Q}_{K}\right)$. This clearly proves that, provided the optimality of the optimization algorithm given in the previous section, there exists a unique Nash equilibrium. It turns out that [17] also allow us to insure the convergence of the proposed algorithm towards the unique maximum, under

\footnotetext{
${ }^{2}$ See also reference [18] which is incomplete but in English.
}

a quite general but non-explicit condition. In order to provide explicit conditions for the existence and uniqueness of a Nash equilibrium we prefer to provide another type of proof. For determining the condition for existence and uniqueness of Nash equilibrium define

$$
g(\alpha) \triangleq \frac{K n_{t}}{n_{r}} \alpha+\frac{1}{K} \sum_{\ell=1}^{K} \int_{\frac{\mu_{\ell}}{\rho \alpha}}^{+\infty}\left(1-\frac{\mu_{\ell}}{\rho \alpha t}\right) f_{\ell}(t) d t-1
$$

Note that if $g$ is strictly decreasing or increasing there is at most one point where it vanishes. We want to show that $\frac{\partial g}{\partial \alpha}$ keeps the same sign on the interval $\left[\alpha_{\min }, \alpha_{\max }\right]$ where $\alpha_{\text {min }}=\frac{n_{r}}{K n_{t}} \frac{1}{1+\rho}, \alpha_{\max }=\frac{n_{r}}{K n_{t}}$. We have

$$
\begin{aligned}
\frac{\partial g}{\partial \alpha}= & \frac{K n_{t}}{n_{r}}+\lim _{T \rightarrow \infty} \frac{1}{K} \sum_{\ell=1}^{K}\left(1-\frac{\mu_{\ell}}{\rho \alpha T}\right) f_{\ell}(t) \frac{\partial T}{\partial \alpha} \\
& -\left(1-\frac{\mu_{\ell}}{\rho \alpha} \frac{\rho \alpha}{\mu_{\ell}}\right) p_{\ell}\left(\frac{\mu_{\ell}}{\rho \alpha}\right) \frac{\partial}{\partial \alpha}\left(\frac{\mu_{\ell}}{\rho \alpha}\right) \\
= & \frac{K n_{t}}{n_{r}}+\frac{1}{K \rho \alpha^{2}} \sum_{\ell=1}^{K} \mu_{\ell} \int_{\frac{\mu_{\ell}}{\rho \alpha}}^{+\infty} \frac{f_{\ell}(t)}{t} d t .
\end{aligned}
$$

We see that $\frac{\partial g}{\partial \alpha}$ is strictly positive on $[0,+\infty)$. This unconditionally insures the uniqueness of the point $\alpha_{0}$ such that $g\left(\alpha_{0}\right)=1$. However this point will not always be in the operating interval $\left[\alpha_{\min }, \alpha_{\max }\right]$. As for all $\alpha \leq 0$, $g(\alpha) \geq \frac{K n_{t}}{n_{r}} \alpha-1$ it is clear that $\alpha_{0} \leq \alpha_{\max }$. Therefore a necessary and sufficient condition for the existence of a Nash equilibrium follows:

$$
\alpha_{0} \geq \frac{n_{r}}{K n_{t}} \frac{1}{1+\rho} .
$$

If this condition is met there exists a unique Nash equilibrium and, in this case we note: $\alpha_{0}=\alpha^{*}$. The main issue here is that it is not easy to provide a closed-form expression for $\alpha_{0}$. This is why determining sufficient but not necessary conditions for the existence of $\alpha^{*}$ turns out to be useful.

For all $\alpha \geq 0$ we have

$$
g(\alpha) \leq \frac{K n_{t}}{n_{r}} \alpha-1+\frac{1}{K} \sum_{\ell=1}^{K} \int_{\frac{\mu_{\ell}}{\rho \alpha}}^{+\infty} f_{\ell}(t) d t .
$$

Let us denote by $\tilde{\alpha}_{0}$ the point where the upper bound function, says $\tilde{g}(\alpha)$, vanishes. By construction $\tilde{\alpha}_{0} \leq \alpha_{0}$. Therefore, from equation (27) we know that if $\tilde{\alpha}_{0} \geq \frac{n_{r}}{K n_{t}} \frac{1}{1+\rho}$ then the existence of a Nash equilibrium is insured. This means that if one can explicit the function

$$
I_{\ell}: \mid \begin{array}{ccc}
{[0,+\infty)} & \rightarrow & {[0,1]} \\
\alpha & \mapsto \int_{\frac{\mu_{\ell}}{\rho \alpha}}^{+\infty} f_{\ell}(t) d t
\end{array}
$$

one can find an explicit sufficient condition for a Nash equilibrium of the multiple access system under consideration.

In what follows we provide sufficient conditions for a typical and realistic antenna correlation profile:

$$
\forall(i, j) \in\left\{1, \ldots, n_{t}\right\}^{2}, T_{k}(i, j)=r_{k}^{|i-j|}
$$

where $r_{k}$ is the correlation coefficient characterizing the transmit correlation matrix $\mathbf{T}_{k}$ (this is also the case for the simulation results provided in sec. 5). The authors of [19] have shown that the asymptotic probability density function of 
the eigenvalues of $\mathbf{T}_{k}$ are simply given by:

$\forall k \in\{1, \ldots, K\}, f_{k}(t)=\mid \begin{array}{ll}\frac{1}{\pi t \sqrt{-t^{2}+2 a_{k} x-1}} & \text { if } \frac{1-r_{k}}{1+r_{k}}<t<\frac{1+r_{k}}{1-r_{k}} \\ 0 & \text { otherwise }\end{array}$

with $a_{k} \triangleq \frac{1+r_{k}^{2}}{1-r_{k}^{2}}$. The corresponding cumulative distribution function is:

$$
F_{k}(t)=\frac{1}{\pi} \cos ^{-1}\left(\frac{1-r_{k}^{2}}{2 r_{k} t}-\frac{1+r_{k}^{2}}{2 r_{k}}\right)
$$

Assuming the chosen correlation profile we find that

$$
I(\alpha)=\mid \begin{array}{cl}
0 & \text { if } \alpha<\frac{\mu_{\ell}}{K \rho} \frac{1-r_{\ell}}{1+r_{\ell}} \\
1-\frac{1}{\pi} \cos ^{-1}\left(A_{\ell}(\alpha)\right) & \text { if } \alpha \in\left[\frac{\mu_{\ell}}{K \rho} \frac{1-r_{\ell}}{1+r_{\ell}}, \frac{\mu_{\ell}}{K \rho} \frac{1+r_{\ell}}{1-r_{\ell}}\right] \\
1 & \text { if } \alpha>\frac{\mu_{\ell}}{K \rho} \frac{1+r_{\ell}}{1-r_{\ell}}
\end{array}
$$

with $A_{\ell}(\alpha)=\frac{K \rho\left(1-r_{\ell}^{2}\right)}{\mu_{\ell} 2 r_{\ell}} \alpha-\frac{1+r_{\ell}^{2}}{2 r_{\ell}}$. By upper bounding the function $I_{\ell}$ by 1 on the interval $\left[\frac{\mu_{\ell}}{K \rho} \frac{1-r_{\ell}}{1+r_{\ell}}, \frac{\mu_{\ell}}{K \rho} \frac{1+r_{\ell}}{1-r_{\ell}}\right]$ one defines a new upper bound function $\hat{g}(\alpha) \geq \tilde{g}(\alpha) \geq g(\alpha)$ that vanishes at a unique point called $\hat{\alpha}_{0}$. This function is defined by

$$
\hat{g}(\alpha)=\frac{K n_{t}}{n_{r}}-1+\frac{1}{K} \sum_{\ell=1}^{K} \mu_{\ell} u\left(\alpha-\frac{\mu_{\ell}}{K \rho} \frac{1-r_{\ell}}{1+r_{\ell}}\right)
$$

where the function $u($.$) is the Heaviside step function. One$ can show that the corresponding nulling point expresses by:

$$
\hat{\alpha}_{0}=\frac{n_{r}}{K n_{t}} \times\left(1-\frac{1}{K} \sum_{\ell=1}^{\hat{K}} \mu_{\ell}\right)
$$

where $\hat{K}$ is the greatest integer in the set $\{1, \ldots, K\}$ such that $1-\frac{K n_{t}}{n_{r}} \alpha_{1}-\frac{1}{K} \sum_{\ell=1}^{\hat{K}} \mu_{\ell} \geq 0$ and the user indices have been ordered so that $\mu_{1} \leq \mu_{2} \leq \ldots \leq \mu_{\ell}$. Thus a sufficient condition for the existence of a Nash equilibrium when the exponential correlation profile (30) is assumed is

$$
\frac{1}{1+\rho} \leq 1-\frac{1}{K} \sum_{\ell=1}^{\hat{K}} \mu_{\ell}
$$

This equation can be implemented and allows each mobile station to insure the existence of a Nash equilibrium. We see that the existence of such an equilibrium tends to be guaranteed as the signal-to-noise ratio increases. Note that in some special cases, like the low and high signal-to-noise ratio (SNR) regimes, the vanishing point of $g(\alpha)$ can be determined exactly whatever the assumed correlation profile:

$$
\left\{\begin{array}{cll}
\rho \rightarrow \infty & : & \alpha^{*} \rightarrow 0 \\
\rho \rightarrow 0 & : & \alpha^{*} \rightarrow \frac{n_{r}}{K n_{t}} \\
K \rightarrow \infty & : & \alpha^{*} \rightarrow 0
\end{array}\right.
$$

\section{SIMULATION RESULTS}

In this section we compare the performance of the proposed distributed power allocation scheme with the uniform power allocation scheme (over the different transmit antennas). First we show that the asymptotic regime is reached for relatively small numbers of antennas. Figure 2 represents the relative error (in percentage) between the maximized ergodic mutual information and its approximation versus signal-to-noise ratio for one user and different correlation scenarios: $\rho=0.2, \rho=0.5$ and $\rho=0.8$ (the transmit antenna correlation profile is the one used in section 4.3), with $n_{t}=n_{r}=4$. It is seen that even in this case, in the considered range of SNR values, the approximation error never exceeds $1 \%$. We then consider the single-user case and compare the performance of uniform and optimal power allocations schemes. Corresponding results are represented in figures 3 and 4 with different values for the correlation parameter $(\rho=0.5$ and $\rho=0.8)$. The gain of the proposed algorithm is quite significant for low SNRs but vanishes as the SNR increases.

We then consider a system with two active users with the following correlation parameters: $\rho_{1}=0.2, \rho_{2}=0.8$. Figure 5 depicts the sum-rate for the uniform and optimal power allocation schemes while figure 6 shows the individual rates for the two users. Interestingly, the optimum power allocation strategy provides significant gains not only for low SNRs but also and especially for high SNRs. It is interesting to note that even at high values of SNR the optimal allocation may result in some (or all) of the users not using all the available dimensions to allocate the power, as can be seen in figure 7 where the powers allocated to each of the eigenvalues are shown. We see that there is a performance saturation for the isotropic power allocation, because in the context considered in this paper (decentralized power allocation with single-user decoding at the base station) the uniform scheme leads to a multiple access interference-limited system whereas the proposed decentralized scheme can cope with the presence of interference. Other simulations results have confirmed this result, which shows the interest of the proposed approach.

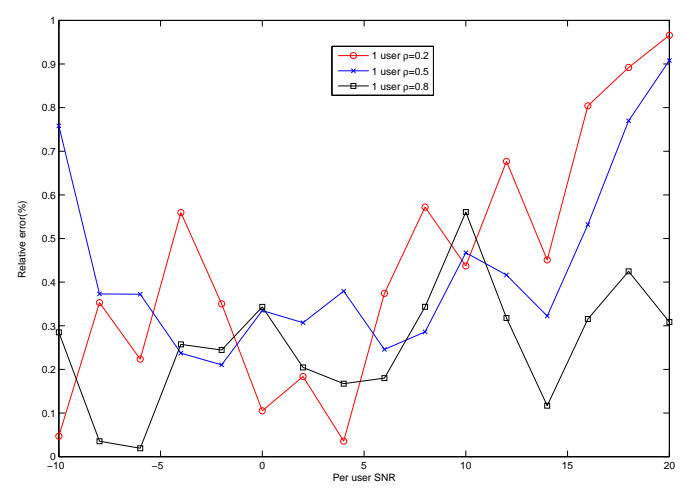

Figure 2: 1 user, $\rho=0.2,0.5,0.8$. Relative error of the asymptotic approximation with respect to actual ergodic capacity. $n_{T}=n_{R}=4$ 


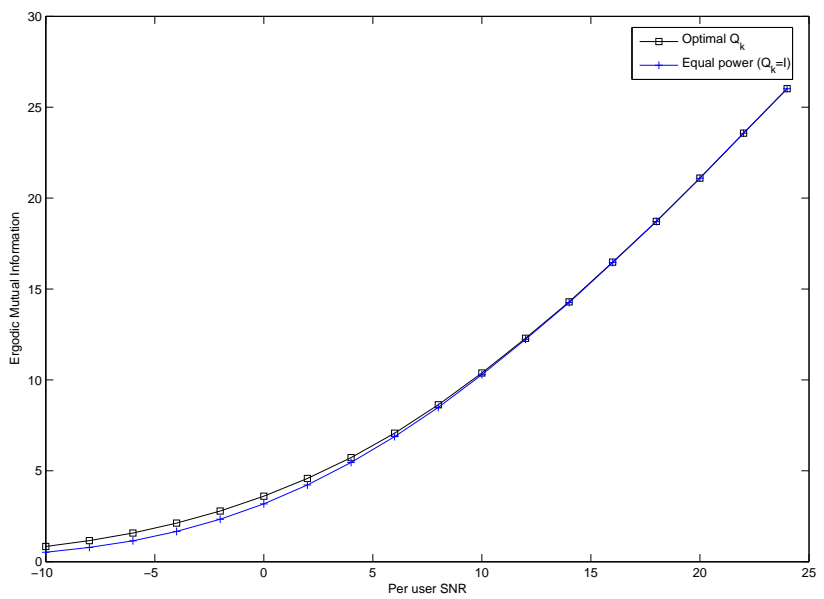

Figure 3: Ergodic mutual information for optimal and insotropic inputs. 1 user, $\rho=0.5, n_{T}=n_{R}=4$.

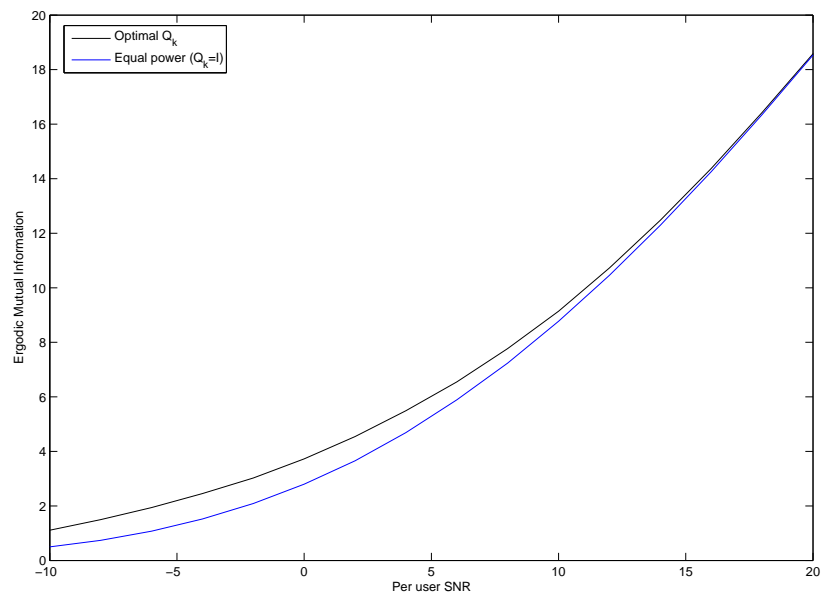

Figure 4: Ergodic mutual information for optimal and insotropic inputs. 1 user, $\rho=0.8, n_{T}=n_{R}=4$.

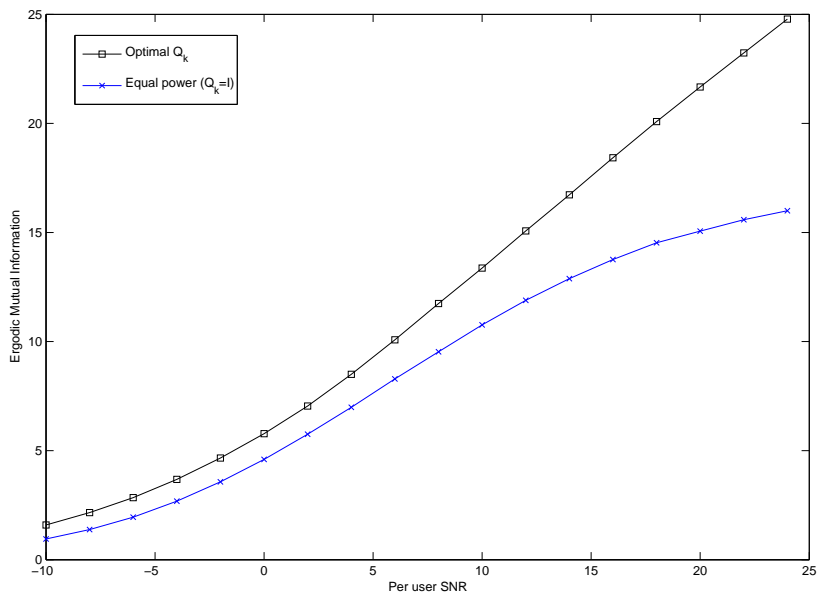

Figure 5: Ergodic sum mutual information for optimal and insotropic inputs. 2 users, $\rho_{1}=0.2, \rho_{2}=0.8$, $n_{T}=n_{R}=4$.

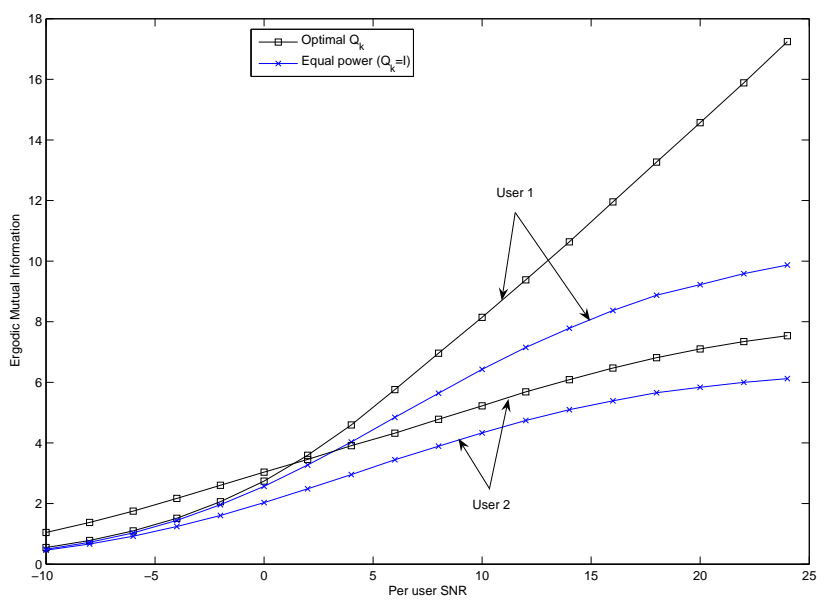

Figure 6: Individual user sum mutual information for optimal and insotropic inputs. 2 users, $\rho_{1}=0.2$, $\rho_{2}=0.8, n_{T}=n_{R}=4$. 


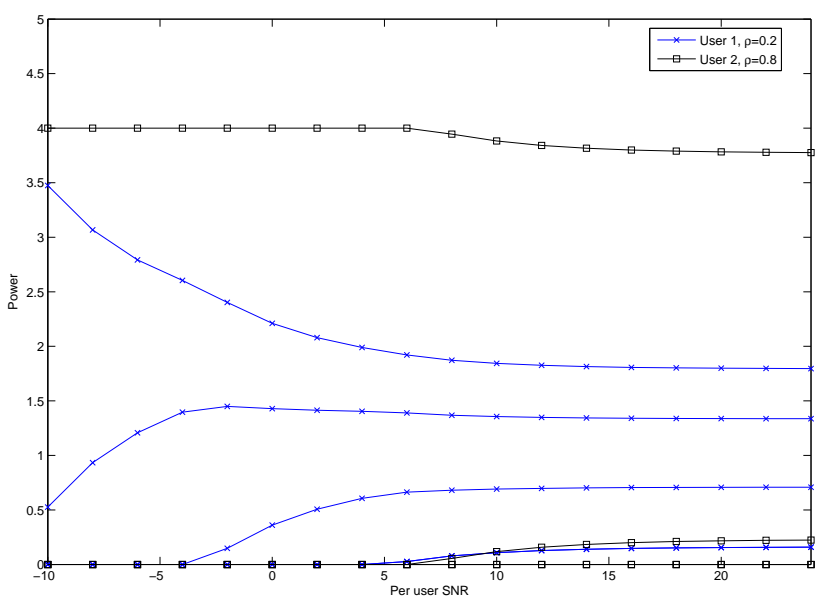

Figure 7: Power allocated to each of the user's 4 eigenmodes. 2 users, $\rho_{1}=0.2$ and $\rho_{2}=0.8, n_{T}=n_{R}=$ 4.

\section{CONCLUSIONS}

In this paper we considered fading MIMO multiple access channels with antenna correlation. We exploited key results from random matrix theory to design a simple decentralized power allocation algorithm. Sufficient conditions for the existence and uniqueness of a Nash equilibrium for the system under investigation have been provided. Simulation results show that the proposed algorithm clearly outperforms the uniform power allocation strategy, because the performance of the latter strategy is severely limited by multiple access interference.

Several interesting extensions of this work could be addressed. First of all, the sufficient conditions for the existence of a Nash equilibrium could be refined. Second, as we consider the individual ergodic mutual information for the user utility function, it would be useful, for slow fading channels, to optimize the outage probability associated with the individual mutual information. Finally, as the sum capacity expression provided by random matrix theory is based on certain fixed parameters (related to the channel statistics) the base station could send a very limited amount of information (which scales properly with the number of users) on these parameters to coordinate the system and therefore obtain a correlated equilibrium aiming at optimizing in a decentralized way the overall system performance [20].

\section{REFERENCES}

[1] D. N. C. Tse and S. Hanly, "Multi-access fading channels - Part I: polymatroid structure, optimal resource allocation and throughput capacities", IEEE Trans. on Inform. Theory, Vol. 44, No. 7, Nov. 1998, pp. 2796-2815.

[2] A. Soysal and S. Ulukus, "Optimum power allocation for single-user MIMO and multi-user MIMO-MAC with partial CSI", IEEE Jour. on Selected Areas in Communications, to appear.
[3] A. Soysal and S. Ulukus, "Optimality of beamforming in fading MIMO multiple access channels", submitted to IEEE Trans. on Communications, January 2007.

[4] D. Fudenberg and J. Tirole, "Game theory", MIT Press, 1991.

[5] E. Altman, T. Boulogne, E. El-Azouzi, T. Jiménez and, L. Wynter, "A survey on networking games in telecommunication", Computer and operations research - Elsevier Science Ltd, Vol. 33, No. 2, pp. 286-311, Feb. 2006.

[6] L. Lai and H. El Gamal, "The water-filling game in fading multiple access channels", submitted to the IEEE Trans. on Inform. Theory, Nov. 2005.

[7] F. Meshkati, H. Poor, S. Schwartz, and N. Mandayam, "An energy-efficient approach to power control and receiver design in wireless data networks", IEEE Trans. on Comm., Vol. 53, No. 11, pp. 1885-1894, Nov. 2005.

[8] F. Meshkati, M. Chiang, H. Poor, and S. Schwartz, "A game-theoretic approach to energy-efficient power control in multi-carrier CDMA systems", IEEE Journal on Selected Areas in Communications, Vol. 24, No. 6, June 2006, pp. 1115-1129.

[9] G. Arslan, M.F. Demirkol and Y. Sang, "Equilibrium efficiency improvement in MIMO interference systems: a decentralized stream control approach", Proceedings of the IEEE International Conference on Communications, Istanbul, Turkey, June 11-15, 2006.

[10] A. M. Tulino, A. Lozano and S. Verdu, "Impact of antenna correlation on the capacity of multiantenna channels", IEEE Trans. on Inform. Theory, Vol. 51, No. 7, July 2005, pp. 2491-2509.

[11] D. S. Shiu, G. J. Foschini, M. J. Gans and J. M. Kahn, "Fading correlation and its effects on the capacity of multielement antenna systems", IEEE Trans. on Comm., Vol. 48, No. 3, Mar. 2000, pp. 502-513.

[12] J. Dumont, P. Loubaton and S. Lasaulce, "On the capacity achieving transmit covariance matrices of MIMO correlated Rician channels: a large system approach", IEEE Proceedings of Globecom Technical Conference, San Francisco, CA, USA, 2006.

[13] L. Zheng, D.N. Tse, "Optimal Diversity-Multiplexing Tradeoff in Multiple Antenna Channels", Proc. of Allerton Conf. on Comm., Control and Computing, Monticello, IL, pp. 835-844, Oct. 2001.

[14] A. Tulino and S. Verdu, "Random Matrices and Wireless Communications", Foundations and trends in communications and information theory, NOW, The Essence of Knowledge, 2004.

[15] Jack W. Silverstein and Z.D. Bai, "On the Empirical Distribution of Eigenvalues of a Class of Large Dimensional Random Matrices", Journal of Multivariate Analysis, 1995, vol. 54, pp. 175-192.

[16] V. L. Girko, "Theory of Stochastic Canonical Equations", Kluwer, 2001, Vol. 1 and Vol. 2, Dordrecht, The Netherlands.

[17] J. Dumont, W. Hachem, S. Lasaulce, P. Loubaton and J. Najim, "Quelques propriétés d'un approximant de l'information mutuelle des canaux MIMO de Rice bi-corrélés", in Proc. of the french Signal and Image Processing Conference (GRETSI), Troyes, France, Sep. 2007. 
[18] J. Dumont, "Optimisation conjointe de l'émetteur et du récepteur par utilisation des a priori du canal dans un contexte MIMO", $\mathrm{PhD}$ dissertation, Université de Marne-la-Vallée, Chapter 1 (in English), Dec. 2006.

[19] A. Skupch, D. Seethaler and F. Hlawatsch, "Free probability based capacity calculation for MIMO channel with transmit or receive correlation", in IEEE Proc. of WirelessComm, June 2005, Hawai, pp. 1041-1046.

[20] E. Altman, N. Bonneau and M. Debbah, "Correlated equilibrium in access control for wireless communications", Networking 2006, Coimbra, Portugal. 